\title{
A Robust aCGH Data Recovery Framework Based on Half Quadratic Minimization
}

\author{
Majid Mohammadia ${ }^{\text {Ghosheh Abed Hodtani*b }}$ \\ ${ }^{a}$ Department of Computer Engineering, Faculty of Engineering, Ferdowsi University of Mashhad, Mashhad, Iran \\ ${ }^{b}$ Department of Electrical Engineering, Faculty of Engineering, Ferdowsi University of Mashhad, Mashhad, Iran
}

\begin{abstract}
This paper presents a general half quadratic framework for simultaneous analysis of the whole array comparative genomic hybridization $(\mathrm{aCGH})$ profiles in a data set. The proposed framework accommodates different M-estimation loss functions and two underlying assumptions for aCGH profiles of a data set: sparsity and low rank. Using M-estimation loss functions, this framework is more robust to various types of noise and outliers. The solution of the proposed framework is given by half quadratic (HQ) minimization. To hasten this procedure, accelerated proximal gradient (APG) is utilized. Experimental results support the robustness of the proposed framework in comparison to the state-of-the-art algorithms.
\end{abstract}

Keywords: Cancer, CNV, aCGH, half quadratic, Correntropy

\section{Introduction}

Finding chromosomal aberrations is an important key to prognosticating and diagnosing various diseases such as cancer. Traditionally, finding these aberrations was accomplished by clinical scientists who were able to detect very little, subtle changes. However, the amount of material aberrations may be too small to be detected by a scientist under a microscope.

Array comparative genomic hybridization (aCGH) is a significant advancement in technology that can detect any small chromosome imbalance that cannot be detected by looking down a microscope. In fact, aCGH is a major approach for discovering copy number variations (CNVs). A CNV is defined as an alteration (gain or loss) in copies of a DNA segment [1].

The main challenge for detecting breakpoints is that aCGH profiles are highly corrupted by large noise and recovering true $\mathrm{CNV}$ s from noisy measurement cannot be done by simply thresholding the raw profiles. As a result, Manifold methods were proposed to recover true aCGH data from noisy measurements.

\subsection{Related Work}

Traditional methods for identifying CNVs include hidden Markov models [2, 3], break point detection $[4,5]$, wavelet transform [6, 7], and variational models [8]. These methods process each aCGH profile for individuals separately. Recently, the simultaneous analysis of whole aCGH profiles has drawn considerable attention.

It is well known that aCGH profiles are corrupted with high level of noise and it is desired to recover the true signal form the observation data. The first model that is exploited is

$$
D=B+E
$$

where $D, B$ and $E$ are $R^{n \times m}$ matrices of the observation data, clean data and noise respectively, $m$ is the number of probes, $n$ is the number of samples and $D_{i j}$ represents the log ratio of $i$-th probe of $j$-th sample.

${ }^{*}$ Corresponding Author; Email: Hodtani@um.ac.ir; Address: Room 848, Faculty of Engineering, Ferdowsi University of Mashhad, Azadi Square, Mashhad, Iran; Postal Code: 9177948944 ; Telephone: +985138805054. 
Based on the model in Eq. (1) and the assumptions that the true aCGH profiles are sparse and piecewise continuous, the following minimization for recovering aCGH data is proposed [9]

$$
\min _{B_{j}}\left\|B_{j}-D_{j}\right\|_{2}^{2}+\lambda_{1}\left\|B_{j}\right\|_{1}+\lambda_{2}\left\|B_{j}\right\|_{T V}
$$

where $\lambda_{1}, \lambda_{2} \geq 0$ are regularization parameters, $B_{j}$ is the $\mathrm{j}$-th column of matrix $B$ and $\|.\|_{2},\|.\|_{1}$ and $\|.\|_{T V}$ are $L_{2}, L_{1}$ and total variation norms, respectively. It is obvious that this method processes each sample separately, but it must be mentioned that it can be easily converted to analyze multisample profiles simultaneously. Another work based on model (1) can be found in [10]. Instead of finding a sparse model of each sample individually, it is supposed that the noise-free aCGH profiles in a data set are linearly correlated so that they can be recovered by finding the low rank approximation of the whole matrix [10]

$$
\min _{B}\|B-D\|_{F}^{2}+\lambda_{1}\|B\|_{*}+\lambda_{2} \sum_{j}\left\|B_{j}\right\|_{T V}
$$

where $\lambda_{1}, \lambda_{2} \geq 0$ are regularization parameters, $\|.\|_{*}$ is nuclear norm, which is defined as the sum of singular values and $\|.\|_{F}$ is Frobenius norm. This method shows excellent performance, especially when the data share a similar pattern. By the same token, the method presented in [11] took into account the individualspecific variations by imposing sparse assumptions on noise and tried to find the decomposition in model (1) by solving the following minimization

$$
\min _{B, E}\|D-B-E\|_{F}^{2}+\lambda_{1}\|B\|_{*}+\lambda_{2} \sum_{j=1}^{n}\left\|B_{j}\right\|_{T V}+\beta\|E\|_{1}
$$

where $\lambda_{1}, \lambda_{2}, \beta \geq 0$ are regularization parameters. Other than the model (1), there exists another model which is based on latent features, i.e.

$$
D=U V+E
$$

where $U \in R^{n \times j}, V \in R^{j \times m}$ and $j$ is the number of latent features. In fact, it is assumed that all samples in a data set can be restated as a weighted combination of several fixed features. In other words, the latent features $U$ can be thought of as common ingredients and weights $V$ are the recipe that determines the composition of each feature to create $D$ [12]. Based on this model, the following minimization was proposed

$$
\begin{aligned}
& \min _{U, V}\|D-U V\|_{F}^{2}+\lambda_{1}\|U\|_{1}+\lambda_{2} \sum_{j}\left\|U_{j}\right\|_{T V} \\
& \text { s.t. }\left\|V_{j}\right\|_{2}^{2} \leq 1
\end{aligned}
$$

where $\lambda_{1}, \lambda_{2} \geq 0$ are regularization parameters. One of the drawbacks of (6) is that it is non-convex over joint $U$ and $V$, but it is convex over $U$ and $V$ separately. After solving (6), the true aCGH profiles will be recovered by multiplying $U$ and $V$.

\subsection{Contribution}

The underlying assumption of all aforementioned methods is that the noise by which aCGH profiles are contaminated is Gaussian. However, this assumption is not valid in all situations and real world applications.

First, we propose a framework based on model (1) for recovering multisample profiles which is robust to various types of noise and predicated on piecewise continuity, sparsity and low rank of the true aCGH signals. The general robust framework for aCGH data recovery is

$$
\min _{B} \sum_{i, j} \phi\left(D_{i j}-B_{i j}\right)+\lambda_{1} F(B)+\lambda_{2} \sum_{j}\left\|B_{j}\right\|_{T V}
$$

where $\lambda_{1}, \lambda_{2} \geq 0$ are regularization parameters, $\phi($.$) is a (convex or non-convex) robust M-estimator which$ can be solved efficiently via half quadratic (HQ) optimization and $F(B)$ can be either $\|B\|_{*}$ or $\|B\|_{1}$. We will investigate a general half quadratic framework to minimize the problem (7). Based on this framework, the 
problem (7) will be reduced to solving a number of unconstrained quadratic problems iteratively.

Second, to increase the speed of convergence, accelerated proximal gradient (APG) [13] is utilized. It will be shown that by utilizing APG, the multiplicative form of the HQ can be restated as the additive form. Finally, different loss functions $\phi($.$) are studied in detail.$

Organization: The rest of the paper is organized as follows. In Section II, half quadratic programming and accelerated proximal gradient (APG) are reviewed. The solution to the proposed method, parameter selection and possible loss functions for $\phi($.$) are brought in Section III. Section IV is dedicated to experimental$ results on real and synthesized data and we conclude the paper in Section V.

\section{Background}

\subsection{Half-Quadratic Minimization}

Before going any further, we review the half-quadratic theory upon which our framework will be proposed. $\mathrm{HQ}$ is predicated on conjugate function theory $[14,15]$ for the convex and non-convex optimization. For a more thorough review, readers are referred to $[16,17]$.

\subsubsection{Half-Quadratic Optimization}

Consider the following minimization problem

$$
\min _{v} \sum_{j=1}^{n} \phi\left(v_{j}\right)+J(v)
$$

where $\phi($.$) is a robust M-estimator and J($.$) is a convex function on v$. In the HQ programming, the loss function $\phi\left(v_{j}\right)$ in (8) can be restated by

$$
\min _{v, p} \sum_{j} Q\left(v_{j}, p_{j}\right)+\psi\left(p_{j}\right)+J(v)
$$

where $p_{j}$ is an auxiliary variable which is determined by a minimization function $\delta(),. Q(.,$.$) is the HQ$ function which has additive and multiplicative forms and $\phi\left(p_{j}\right)$ is the convex conjugate of loss function $\phi($.$) and defined as [18]$

$$
\psi(p)=\max _{s} \quad\left(p^{T} s-\phi(s)\right) .
$$

The function $\psi(p)$ is convex since it is the point-wise maximum of convex functions of $p$ [18], therefore, problem (9) is convex and can be iteratively minimized as

$$
\begin{aligned}
& p^{k+1}=\delta\left(v^{k}\right) \\
& v^{k+1}=\arg \min _{v} Q\left(v, p^{k+1}\right)+J(v)
\end{aligned}
$$

where $k$ denotes the iteration and $\delta($.$) is the minimizer function which is only related to \phi($.$) .$

\subsubsection{The Additive and Multiplicative Forms}

To utilize HQ minimization, there exist two quadratic forms for $Q\left(v_{j}, p_{j}\right)$ : Additive and Multiplicative denoted by $Q_{A}\left(v_{j}, p_{j}\right)$ and $Q_{M}\left(v_{j}, p_{j}\right)$, respectively $[14,15]$. Specifically, $Q_{A}\left(v_{j}, p_{j}\right)$ can be reformulated as [15]

$$
Q_{A}\left(v_{j}, p_{j}\right)=\left(v_{j}-p_{j}\right)^{2} .
$$

The additive form indicates that we can restate $\phi($.$) as a combination of quadratic terms and the auxiliary$ variable $p$.

The multiplicative form for $Q_{M}\left(v_{j}, p_{j}\right)$ is formulated in the form of

$$
Q_{M}(v, p)=\frac{1}{2} p_{j} v_{j}^{2}
$$


This form is usually compared with the reweighted least square problem.

In [16], it is shown that when the additive form is applied, the number of iterations for convergence is greater than the number of iterations of the multiplicative form. In each iteration, however, the additive form has lower computational cost. Overall, optimization by the additive form takes less time than the multiplicative form in real applications [16]. The additive form has drawn less attention despite the fact that it is more economical in computation. In the next section, we give an efficient solution to the minimization (7) with both additive and multiplicative forms for $\phi($.$) .$

\subsection{Accelerated Proximal Gradient}

To speed up the convergence of any (smooth and non-smooth) convex programming, the accelerated proximal gradient (APG) method was proposed based on the first order approximation [13, 19]. According to the APG method, for an arbitrary differentiable convex function $f(x)$ with Lipschitz continuous gradient,

$$
\left\|\nabla f\left(x_{1}\right)-\nabla f\left(x_{2}\right)\right\|_{F} \leq L_{f}\left\|x_{1}-x_{2}\right\|_{F},
$$

and any convex, possibly not differentiable, function $g($.$) , the minimization of objective function$

$$
F(x)=f(x)+g(x)
$$

can be dramatically improved to $O\left(k^{-2}\right)$, where $k$ is the number of iterations.

APG finds the optimal solution of $F(x)$ by its first order approximation at a point $y$ as

$$
Q(x, y)=f(y)+\langle\nabla f(y), x-y\rangle+\frac{L_{f}}{2}\|x-y\|_{F}^{2}+g(x) .
$$

Moreover, it was shown that for any $y, Q(x, y)$ is an upper bound for $F(x)$ so that $x$ is a unique minimizer [13]. Also, $x$ can be updated as

$$
\begin{aligned}
x_{k} & =\arg \min _{x} Q\left(x, y_{k}\right) \\
& =\arg \min _{x} g(x)+\frac{L_{f}}{2}\left\|x-z_{k}\right\|_{F}^{2}
\end{aligned}
$$

where $z_{k}=y_{k}-\frac{1}{L_{f}} \nabla f\left(y_{k}\right)$.

It is also investigated that for any $t_{k}$ satisfying $t_{k}^{2}-t_{k}=t_{k-1}^{2}$, choosing

$$
y_{k}=x_{k}+\frac{t_{k-1}-1}{t_{k}}\left(x_{k}-x_{k-1}\right)
$$

can get the convergence rate of $O\left(k^{-2}\right)[13]$.

\section{Materials and Methods}

In this section, the solution to the proposed framework (7) is given. This is followed by various Mestimators for $\phi($.$) and finding tuning parameters \lambda_{1}$ and $\lambda_{2}$ in (7).

\subsection{Robust Half Quadratic-based aCGH Data Analysis}

To find the solution of the problem (7), it must be rewritten similar to Eq. (9), i.e.

$$
\min _{B, E} \sum_{i, j} Q\left(B_{i j}-D_{i j}, E_{i j}\right)+\psi\left(E_{i j}\right)+\lambda_{1} F(B)+\lambda_{2} \sum_{j}\left\|B_{j}\right\|_{T V}
$$

where $E$ is an auxiliary variable which is determined by the HQ procedure. Let $\sum_{i, j} Q\left(B_{i j}-D_{i j}, E_{i j}\right)+$ $\psi\left(E_{i j}\right)=Q(B-D, E)+\psi(E)$, it is obvious that the problem (17) is equivalent to the following minimization

$$
\begin{array}{ll}
\min _{B, E} & Q(B-D, E)+\psi(E)+\lambda_{1} F(B)+\lambda_{2} \sum_{j}\left\|Z_{j}\right\|_{T V} \\
\text { s.t. } & B=Z .
\end{array}
$$


The augmented Lagrangian of (18) is

$$
\mathcal{L}=Q(B-D, E)+\psi(E)+\lambda_{1} F(B)+\lambda_{2} \sum_{j}\left\|Z_{j}\right\|_{T V}+<Y, B-Z>+\frac{\mu}{2}\|B-Z\|_{F}^{2}
$$

where $Y$ is the Lagrangian multiplier and $\mu>0$ is a positive constant. Then, the following steps must be iterated until convergence

$$
\begin{aligned}
& E_{k+1}=\arg \min _{E} Q\left(B_{k}-D, E\right)+\psi(E) \\
& B_{k+1}=\arg \min _{B} \lambda_{1} F(B)+Q\left(B-D, E_{k+1}\right)+\frac{\mu}{2}\left\|B-Z_{k}+\frac{Y_{k}}{\mu}\right\|_{F}^{2} \\
& Z_{k+1}=\arg \min _{Z} \lambda_{2} \sum_{j}\left\|Z_{j}\right\|_{T V}+\frac{\mu}{2}\left\|B_{k+1}-Z_{k}+\frac{Y_{k}}{\mu}\right\|_{F}^{2} .
\end{aligned}
$$

To hasten the speed of convergence, the above steps are rewritten by accelerated proximal gradient. Thus, we utilize Eq. (15) and let $L_{f}=2$, we have

$$
\begin{aligned}
& E_{k+1}=\arg \min _{E}\left\|E-G_{k}^{E}\right\|_{F}^{2}+\psi(E) \\
& B_{k+1}=\arg \min _{B} \lambda_{1} F(B)+\left\|B-G_{k}^{B}\right\|_{F}^{2} \\
& Z_{k+1}=\arg \min _{Z} \lambda_{2} \sum_{j}\left\|Z_{j}\right\|_{T V}+\left\|Z-G_{k}^{Z}\right\|_{F}^{2}
\end{aligned}
$$

where

$$
\begin{aligned}
G_{k}^{B} & =Y_{k}^{B}-\frac{1}{2} \nabla_{B}\left(Q\left(D-Y_{k}^{B}, Y_{k}^{E}\right)+\frac{\mu}{2}\left\|Y_{k}^{B}-Z_{k}^{B}+\frac{Y_{k}}{\mu}\right\|_{F}^{2}\right) \\
G_{k}^{E} & =Y_{k}^{E}-\frac{1}{2} \nabla_{E}\left(Q\left(D-Y_{k}^{B}, Y_{k}^{E}\right)\right) \\
G_{k}^{Z} & =Y_{k}^{Z}-\frac{1}{2}\left(Y_{k}^{Z}-Y_{k}^{B}-\frac{Y_{k}}{\mu}\right)
\end{aligned}
$$

and $y_{k}$ for each variable is calculated by (16).

Next the solution to each step is given. The auxiliary variable E in Eq. (21) is determined by minimizer function $\delta($.$) which is only related to the loss function \phi($.$) (see Table 1). The B-step in (22) depends on the$ function $F(B)$ :

- If $F(B)=\|B\|_{*}$, then

$$
B_{k+1}=S V T_{\frac{\lambda_{1}}{2}}\left(G_{k}^{B}\right)
$$

where $S V T_{\alpha}(X)$ means the SVT operator [20]

$$
S V T_{\alpha}(X)=U \Sigma_{\alpha} V
$$

$\Sigma_{\alpha}=\operatorname{diag}\left[\left(d_{1}-\alpha\right)_{+}, \ldots .,\left(d_{l}-\alpha\right)_{+}\right], U \Sigma V$ is singular value decomposition and $(z)_{+}=\max (z, 0)$.

- If $F(B)=\|B\|_{1}$, then

$$
B_{k+1}=S_{\frac{\lambda_{1}}{2}}\left(G_{k}^{B}\right)
$$

where $S_{\beta}(R)_{i j}=\operatorname{sign}\left(R_{i j}\right)\left(R_{i j}-\beta\right)_{+}$refers to the element-wise soft thresholding operator [21].

The Z-step in Eq. (23) can be optimized column-wise as

$$
Z_{j}=\arg \min _{p}\|p\|_{T V}+\left\|p-\left(G_{k}^{Z}\right)_{j}\right\|_{2}^{2}
$$

which is fused lasso signal approximation (FLSA) and can be solved by [12]. Algorithm 1 summarizes the procedure of recovering aCGH data based on half quadratic minimization.

As $\mu_{k}$ converges to $\hat{\mu}>0$, the proof of convergence of Algorithm 1 becomes identical to [22] and [23]. According to [23], $\mu$ oscillates from a big initial value $\mu_{0}$ and diminishes until it reaches the minimum $\hat{\mu}$ and $\theta$ and $\eta$ are set to $10^{-8}$ and 0.9 respectively. 


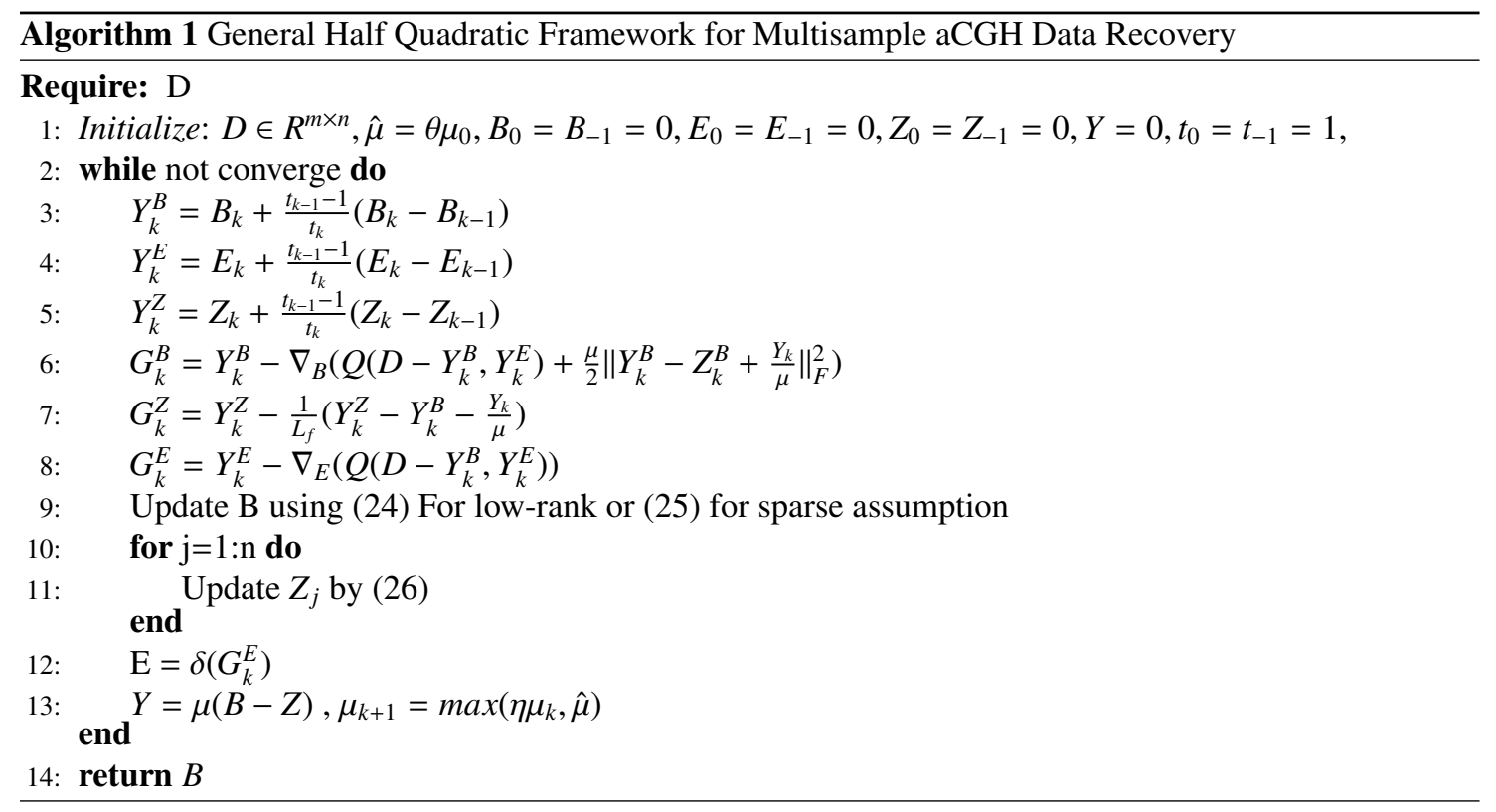

\subsection{Parameter Tuning}

The methodology, by which the parameters $\lambda_{1}$ and $\lambda_{2}$ are determined, is explored in this section. First, the data set is separated into subsets of $S 1$ and $S 2$. Let $P_{\gamma}($.$) be the projection operator by definition$

$$
P_{\gamma}(X)(i, j)= \begin{cases}X_{i j} & \text { if }(i, j) \in \gamma \\ 0 & \text { otherwise }\end{cases}
$$

then, we apply the proposed method on the $S_{1}$ subset with different values for $\lambda_{1}$ and $\lambda_{2}$ :

$$
\left.\min _{B} \phi\left(P_{S_{1}}(D)-B\right)\right)+\lambda_{1} F(B)+\lambda_{2} \sum_{j}\left\|B_{j}\right\|_{T V} .
$$

Assume that the solution of $(27)$ is $\hat{B}\left(\lambda_{1}, \lambda_{2}\right)$. Next, the best $\left(\lambda_{1}, \lambda_{2}\right)$ will be chosen, i.e. $\left(\lambda_{1}, \lambda_{2}\right)$ by which the following prediction error would be minimized

$$
\operatorname{Err}\left(\lambda_{1}, \lambda_{2}\right)=\frac{1}{2}\left\|P_{S_{2}}(D)-\hat{B}\left(\lambda_{1}, \lambda\right)\right\|_{F}
$$

As the search in 2D grid is computationally expensive, we reparameterize $\lambda_{1}=\alpha \lambda_{0}$ and $\lambda_{2}=(1-\alpha) \lambda_{0}$ similar to [12]. We fixed the possible values for $\alpha$ as $\{0.1,0.2, \ldots, 0.9\}$ and then found the value for $\lambda_{0}$ in $[0.1,5]$ which has the minimum value for prediction error (28). After choosing $\lambda_{0}$ and $\alpha$, the parameters $\lambda_{1}$ and $\lambda_{2}$ can be obtained.

\subsection{M-estimation For Large Corruption}

In this section, we focus on the possible loss functions of $\phi($.$) . In robust statistics, a robust technique is M-$ estimation [24]. Table 1 tabulates different M-estimation loss functions and their corresponding minimizer functions $\delta($.$) for the additive form of \phi($.$) . As we utilized the APG method in (21), it is readily seen that$ the minimizer function to find $E$ must be additive, whether we use the additive or multiplicative form for $Q\left(.\right.$, .). But, $G^{E}$ in (21) has different values for the additive and multiplicative forms.

In information theoretic learning (ITL), the robustness of Correntropy [25, 26, 27] and Renyi's quadratic entropy [28] is scrutinized by their relation with the Welsch M-estimator. The probabilistic meaning of Correntropy is that it maximizes the error probability density at the origin [25]. This property guarantees the theoretical support that Correntropy can deal with large corruption. 
Huber M-estimation is another possible function for $\phi($.$) which its affinity with sparse representation is$ explored in [29]. Let us choose Huber loss function for $\phi($.$) in (7) and F(B)=\|B\|_{*}$, thus

$$
\min _{E, B}\|D-B-E\|_{F}^{2}+\psi(E)+\lambda_{1}\|B\|_{*}+\lambda_{2} \sum_{j=1}^{n}\left\|B_{j}\right\|_{T V} .
$$

It was investigated that convex conjugate $\psi($.$) of Huber loss is \beta\|.\|_{1}$ [29], hence the (29) is equivalent to RPLA (4). Therefore, RPLA is a special case of the proposed framework (7).

It is justified that RPLA is more robust in comparison to other algorithms because it minimizes Huber loss function rather than square error. Correntropy (Welsch M-estimator), on the other hand, has shown better performance in dealing with large corruptions [30, 29]. Our experimental results support that the proposed framework with Correntropy loss function has better performance.

\section{Experimental Results}

In this section, the proposed methods are compared with RPLA [11], TVSP [10] and GFLseg [31]. The Matlab code of all methods are available from the Mat-aCGH toolbox [32]. In Algorithm 1, it is possible to choose either the additive or multiplicative form for function $Q(.$, .). The additive form is chosen because it converges in less time than the multiplicative form [16]. Moreover, the proposed algorithms benefit from the fast convergence of APG so that it has less complexity in each iteration and fast convergence at the same time. For the sake of simplicity, we call the method $\mathrm{LRHQ}^{1}$ when $F(B)=\|B\|_{*}$ and $\mathrm{SHQ}^{2}$ when $F(B)=\|B\|_{1}$.

The Huber estimator, the $l_{1}-l_{2}$ M-estimator and the Welsch M-estimator are mainly used in various experiments. If the Huber M-estimator is employed, the thresholding parameter for $\delta($.$) will be \frac{\mu \lambda}{2}$ and the parameters can be selected exactly like [11]. The parameter $\sigma^{2}$ in the $l_{1}-l_{2}$ and Welsch M-estimators are estimated by the robust mean value as $\sigma^{2}=\operatorname{mean}\left(\left(G_{k}^{E}\right)^{2}\right)[33]$.

\subsection{Simulated Data}

In this subsection, the performance of the proposed framework is compared with other aforementioned algorithms on simulated data. To do so, we generate 50 samples with the length of 500 probes and an arbitrary noise is added to the profiles. Two types of aberrations are considered. The first type is added to each individual sample at a random location independently and the second type is shared aberrations that are presented at the same location of multiple samples. The ratio between shared aberrations and total aberrations is called share percentage [10]. The length of each segment is selected from $\{5,15,30,40,50\}$. To better evaluate various methods on diverse noise, five non-Gaussian noises are added to the simulated data. For each distribution, five random noises are generated which their parameters are randomly selected according to setting in Table 2 .

Table 2: Probability density functions (PDF) and their parameters utilized as an additive noise to simulated data. For generating noise, the parameters are randomly selected in the range tabulated in the last row.

Figure 1: Performance Comparison of LRHQ, SHQ, TVSP [10], RPLA [11] and GFLseg [31]. Each row and each column dedicated to a specific SNR and shared percentage, respectively. The $x$-axis and $y$-axis of each plot represent the false positive rate and true positive rate, respectively. In each case, five randomly generated distributions are generated according to Table 2 and the mean of ROC curve is plotted for each case.

\footnotetext{
${ }^{1}$ Low Rank Half Quadratic-based method

${ }^{2}$ Sparse Half Quadratic-based method
} 
The average of ROC curve for four non-guassian noise with diverse distributions is ploted in Fig 1. In this figure, for each of these distributions the average of ROC over five randomly generated profiles with noise is reported. The ROC curve is created by plotting the true positive rate (TPR) against the false positive rate (FPR) at different threshold setting. TPR and FPR for a threshold $T$ are defined as

$$
\operatorname{TPR}(T)=\frac{\left|P_{T}\right|}{|A P|} \quad F P R(T)=\frac{\left|F P_{T}\right|}{|N|}
$$

where $\left|P_{T}\right|$ and $\left|F P_{T}\right|$ are the numbers of true discovered aberrations and false discovered aberrations with respect to threshold $T,|A P|$ and $|N|$ are number real aberrations and real normal, respectively. As we aim to plot ROC on synthesized data, the real and estimated data are available, therefore, the above-mentioned parameters in TPR and FPR for a threshold $T$ can be easily obtained.

In each case, noise with different SNRs, shared percentages and settings is added and the performance of aforementioned algorithms are compared. As ROC curve deviating from diagonal line, it indicates better performance. From Fig. 1, it can be easily seen that the proposed algorithms outperform other methods in almost all cases and it can be concluded that other methods are not robust when the data are highly corrupted with non-Gaussian noise.

Further, the above methods are compared from the accuracy point of view. The accuracy (ACC) for a threshold $T$ is defined as

$$
\operatorname{ACC}(T)=\frac{\left|\mathcal{N}_{T}\right|+\left|\mathcal{P}_{T}\right|}{|\mathcal{A}|}
$$

where $\left|\mathcal{N}_{T}\right|,\left|\mathcal{P}_{T}\right|$ and $|\mathcal{A}|$ are the number of true discovered normal, true discovered aberration and the total population in regard to threshold $T$, respectively. Five randomly generated distributions for Beta noise with settings tabulated in Table 2 are added to the simulated data. Fig. 2 plots the mean of accuracy of different methods with different thresholds. It is readily seen that the proposed methods achieve a higher accuracy rate in comparison with other approaches.

Figure 2: The accuracy comparison on simulated data corrupted by Beta noise. Five random Beta noises are generated according to setting in Table 2 and the mean of accuracy is reported.

\subsection{Real Data}

Figure 3: Heat and bar diagrams for real data sets. (a) Performing LRHQ on the data set introduced in [34] (b) Performing SHQ on the data set introduced in [35].

We take the Welsch M-estimator as the loss function $\phi($.$) in Algorithm 1$ and apply the proposed methods on two real world data sets, i.e. Pollack et al. [34] and Chin et al. [35]. The first data set has 44 samples and the latter includes 141 profiles of breast tumors. Fig. 3 shows the recovered matrices of chromosome 17 using heat map and bar plot. The first row shows heat and bar diagrams of recovered Pollack et al. data set [34] by LRHQ and the second row tabulates the same diagrams for the recovered Chin et al. data by SHQ.

As the final experiment, several profiles of [34] are chosen and the profiles before and after processing are plotted in Fig. 4.

Figure 4: Array CGH profile of 4 chromosomes of the breast cancer data set [34]. Panels in the same rows show the result of the same chromosome with different methods.

\subsection{Computational Time}

As the APG method is utilized, the convergence rate of the proposed framework is $O\left(k^{-2}\right)$, where k is the number of iteration. Further, when $F(B)=\|B\|_{*}$, the SVT operator must be used which requires the SVD decomposition with order of $O\left(n m^{2}\right)$. In contrast, when $F(B)=\|B\|_{1}$, the Soft Thresholding operator 
is utilized with order of $O(\mathrm{~nm})$. Another step in Algorithm 1 is fused lasso signal approximation which is of order $O(n)$. Overall, the order of LRHQ and SHQ are $O\left(\mathrm{~nm}^{2}\right)$ and $O(\mathrm{~nm})$, respectively. Because the number of probes is much greater than the number of samples, we apply the proposed framework on simulated data with fixed number of samples and different number of probes. In each case, each method is performed twice and the standard deviation is reported. Fig. 5 plots the CPU time against number of probes for different methods. It can be readily seen that the computational time of the proposed methods increase linearly over the number of probes.

Figure 5: CPU time versus number of probes on simulated data with different numbers of probes. The number of samples is 100. For each number of probes, each method is performed twice and the standrad deviation is reported.

\section{Conclusion and Future Work}

This paper presented a robust framework for multisample aCGH data analysis. The proposed framework accommodated the sparsity, low rank and piecewise constancy assumptions of true aCGH signals and tried to recover them accordingly. The proposed problem is not convex, but its efficient solution could be obtained by half quadratic programming and its convergence to a local optimum was guaranteed. In comparison to the state-of-the-art algorithms, the proposed framework demonstrated excellent robustness, especially when data are corrupted with large, non-Gaussian noise.

However, the proposed problem is not smooth and therefore, it oscillates around the optimal solution and converges slowly toward it. To overcome this issue, one can use the $l_{1}-l_{2}$ loss function in Table 1 for $F(B)$ which is smooth and converges faster. Further, the total variation of the whole aCGH profile can be utilized instead of the total variation of each individual sample. These modifications can increase the speed of convergence and the robustness of the algorithm.

\section{Acknowledgement}

This work is supported by the Iranian National Science Foundation (INSF).

\section{Reference}

[1] L. Feuk, A. R. Carson, and S. W. Scherer, "Structural variation in the human genome," Nature Reviews Genetics, vol. 7, no. 2, pp. 85-97, 2006.

[2] S. P. Shah, X. Xuan, R. J. DeLeeuw, M. Khojasteh, W. L. Lam, R. Ng, and K. P. Murphy, "Integrating copy number polymorphisms into array cgh analysis using a robust hmm," Bioinformatics, vol. 22, no. 14, pp. e431-e439, 2006.

[3] S. Guha, Y. Li, and D. Neuberg, "Bayesian hidden markov modeling of array cgh data," Journal of the American Statistical Association, vol. 103, no. 482, pp. 485-497, 2008

[4] P. M. Rancoita, M. Hutter, F. Bertoni, and I. Kwee, "Bayesian dna copy number analysis," BMC bioinformatics, vol. 10, no. 1, p. $10,2009$.

[5] F. Picard, S. Robin, M. Lavielle, C. Vaisse, and J.-J. Daudin, "A statistical approach for array cgh data analysis," BMC bioinformatics, vol. 6, no. 1, p. 27, 2005.

[6] A. Mitra, G. Liu, and J. Song, "A genome-wide analysis of array-based comparative genomic hybridization (cgh) data to detect intra-species variations and evolutionary relationships," PloS one, vol. 4, no. 11, p. e7978.

[7] Y. Wang, S. Wang, and A. R. Zinn, "rswti: A robust stationary wavelet denoising method for array cgh data," in Bioinformatics and Bioengineering, 2007. BIBE 2007. Proceedings of the 7th IEEE International Conference on, pp. 1066-1070, IEEE, 2007.

[8] S. Morganella, L. Cerulo, G. Viglietto, and M. Ceccarelli, "Vega: Variational segmentation for copy number detection," Bioinformatics, vol. 26, no. 24, pp. 3020-3027, 2010

[9] R. Tibshirani and P. Wang, "Spatial smoothing and hot spot detection for cgh data using the fused lasso," Biostatistics, vol. 9, no. 1 , pp. $18-29,2008$.

[10] X. Zhou, C. Yang, X. Wan, H. Zhao, and W. Yu, "Multisample acgh data analysis via total variation and spectral regularization," Computational Biology and Bioinformatics, IEEE/ACM Transactions on, vol. 10, no. 1, pp. 230-235, 2013.

[11] X. Zhou, J. Liu, X. Wan, and W. Yu, "Piecewise-constant and low-rank approximation for identification of recurrent copy number variations," Bioinformatics, p. btu131, 2014.

[12] G. Nowak, T. Hastie, J. R. Pollack, and R. Tibshirani, "A fused lasso latent feature model for analyzing multi-sample acgh data," Biostatistics, p. kxr012, 2011.

[13] A. Beck and M. Teboulle, "A fast iterative shrinkage-thresholding algorithm for linear inverse problems," SIAM Journal on Imaging Sciences, vol. 2, no. 1, pp. 183-202, 2009.

[14] D. Geman and G. Reynolds, "Constrained restoration and the recovery of discontinuities," IEEE Transactions on pattern analysis and machine intelligence, vol. 14, no. 3, pp. 367-383, 1992. 
[15] D. Geman and C. Yang, "Nonlinear image recovery with half-quadratic regularization," Image Processing, IEEE Transactions on, vol. 4, no. 7, pp. 932-946, 1995.

[16] M. Nikolova and M. K. Ng, "Analysis of half-quadratic minimization methods for signal and image recovery," SIAM Journal on Scientific computing, vol. 27, no. 3, pp. 937-966, 2005

[17] R. He, B. Hu, X. Yuan, and L. Wang, Robust recognition via information theoretic learning. Springer, 2014.

[18] S. Boyd and L. Vandenberghe, Convex optimization. Cambridge university press, 2009.

[19] Y. Nesterov, "A method of solving a convex programming problem with convergence rate o (1/k2),"

[20] E. J. Candès, X. Li, Y. Ma, and J. Wright, "Robust principal component analysis?," Journal of the ACM (JACM), vol. 58, no. 3 , p. 11,2011

[21] S. Boyd, N. Parikh, E. Chu, B. Peleato, and J. Eckstein, "Distributed optimization and statistical learning via the alternating direction method of multipliers," Foundations and Trends® in Machine Learning, vol. 3, no. 1, pp. 1-122, 2011.

[22] A. Beck and M. Teboulle, "A fast iterative shrinkage-thresholding algorithm for linear inverse problems," SIAM Journal on Imaging Sciences, vol. 2, no. 1, pp. 183-202, 2009.

[23] R. He, T. Tan, and L. Wang, "Robust recovery of corrupted low-rankmatrix by implicit regularizers," Pattern Analysis and Machine Intelligence, IEEE Transactions on, vol. 36, no. 4, pp. 770-783, 2014

[24] P. J. Huber, Robust statistics. Springer, 2011.

[25] W. Liu, P. P. Pokharel, and J. C. Príncipe, "Correntropy: properties and applications in non-gaussian signal processing," Signal Processing, IEEE Transactions on, vol. 55, no. 11, pp. 5286-5298, 2007.

[26] I. Santamaría, P. P. Pokharel, and J. C. Principe, "Generalized correlation function: definition, properties, and application to blind equalization," Signal Processing, IEEE Transactions on, vol. 54, no. 6, pp. 2187-2197, 2006.

[27] M. Mohammadi, H. S. Noghabi, G. A. Hodtani, and H. R. Mashhadi, "Robust and stable gene selection via maximum-minimum correntropy criterion," bioRxiv, p. 029538, 2015.

[28] J. C. Principe, Information theoretic learning: Rényi's entropy and kernel perspectives. Springer Science \& Business Media, 2010.

[29] R. He, W. Zheng, T. Tan, and Z. Sun, "Half-quadratic based iterative minimization for robust sparse representation,” 2013.

[30] R. He, T. Tan, L. Wang, and W.-S. Zheng, "1 2, 1 regularized correntropy for robust feature selection," in Computer Vision and Pattern Recognition (CVPR), 2012 IEEE Conference on, pp. 2504-2511, IEEE, 2012.

[31] K. Bleakley and J.-P. Vert, "The group fused lasso for multiple change-point detection," arXiv preprint arXiv:1106.4199, 2011.

[32] M. Mohammadi and H. S. Noghabi, "Mat-acgh: a matlab toolbox for simultaneous multisample acgh data analysis and visualization," bioRxiv, p. 028761, 2015.

[33] R. He, W.-S. Zheng, and B.-G. Hu, "Maximum correntropy criterion for robust face recognition," Pattern Analysis and Machine Intelligence, IEEE Transactions on, vol. 33, no. 8, pp. 1561-1576, 2011.

[34] J. R. Pollack, T. Sørlie, C. M. Perou, C. A. Rees, S. S. Jeffrey, P. E. Lonning, R. Tibshirani, D. Botstein, A.-L. Børresen-Dale, and P. O. Brown, "Microarray analysis reveals a major direct role of dna copy number alteration in the transcriptional program of human breast tumors," Proceedings of the National Academy of Sciences, vol. 99, no. 20, pp. 12963-12968, 2002.

[35] K. Chin, S. DeVries, J. Fridlyand, P. T. Spellman, R. Roydasgupta, W.-L. Kuo, A. Lapuk, R. M. Neve, Z. Qian, T. Ryder, et al., "Genomic and transcriptional aberrations linked to breast cancer pathophysiologies," Cancer cell, vol. 10, no. 6, pp. 529-541, 2006. 
Figure 1: Performance Comparison of LRHQ, SHQ, TVSP [10], RPLA [11] and GFLseg [31]. Each row and each column dedicated to a specific SNR and shared percentage, respectively. The $x$-axis and $y$-axis of each plot represent the false positive rate and true positive rate, respectively. In each case, five randomly generated distributions are generated according to Table 2 and the mean of ROC curve is plotted for each case.

(a) Data contaminated with Laplace noise

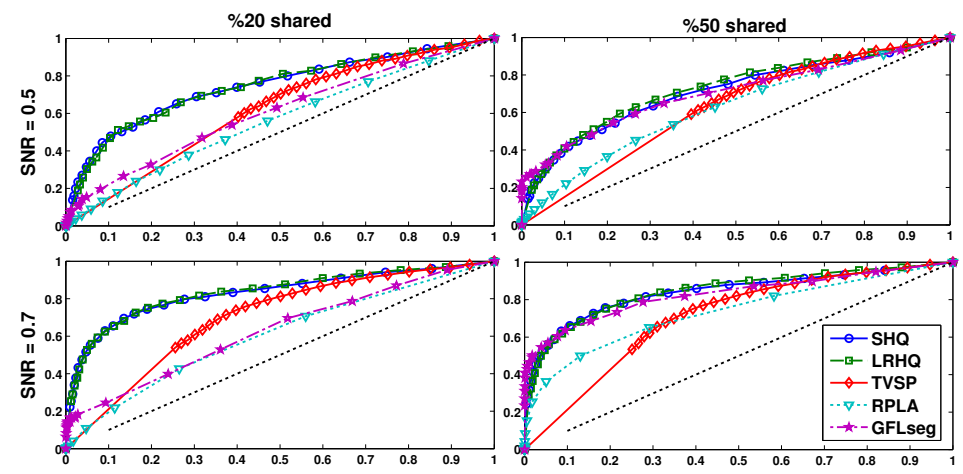

(b) Data contaminated with Exponential noise

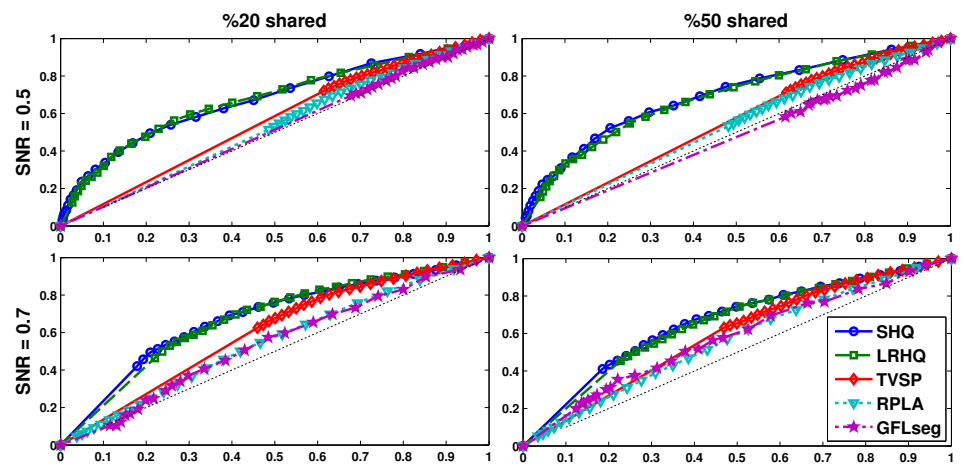

(c) Data contaminated with Gamma noise

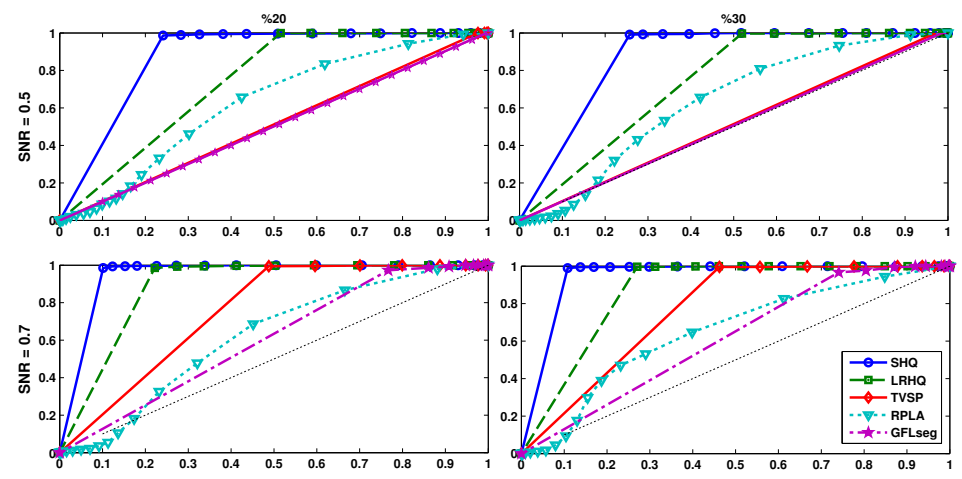

(d) Data contaminated with Uniform noise
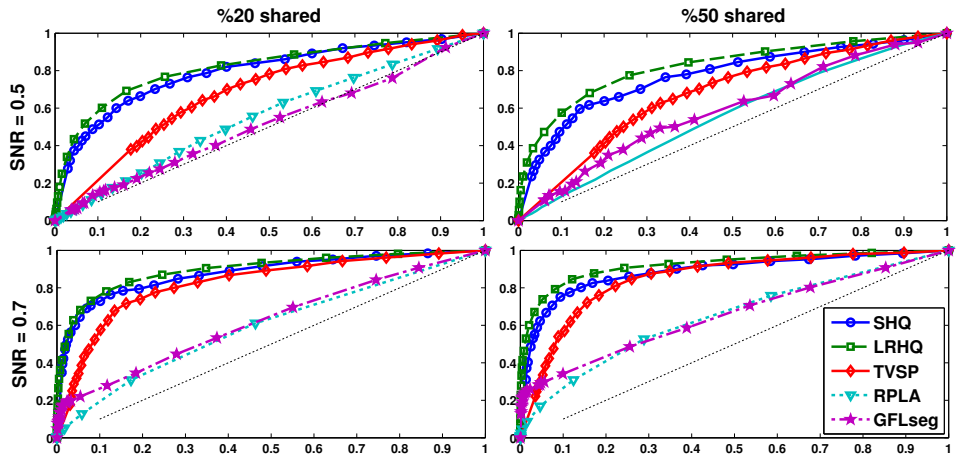
Figure 2: The accuracy comparison on simulated data corrupted by Beta noise. Five random Beta noises are generated according to setting in Table 2 and the mean of accuracy is reported.
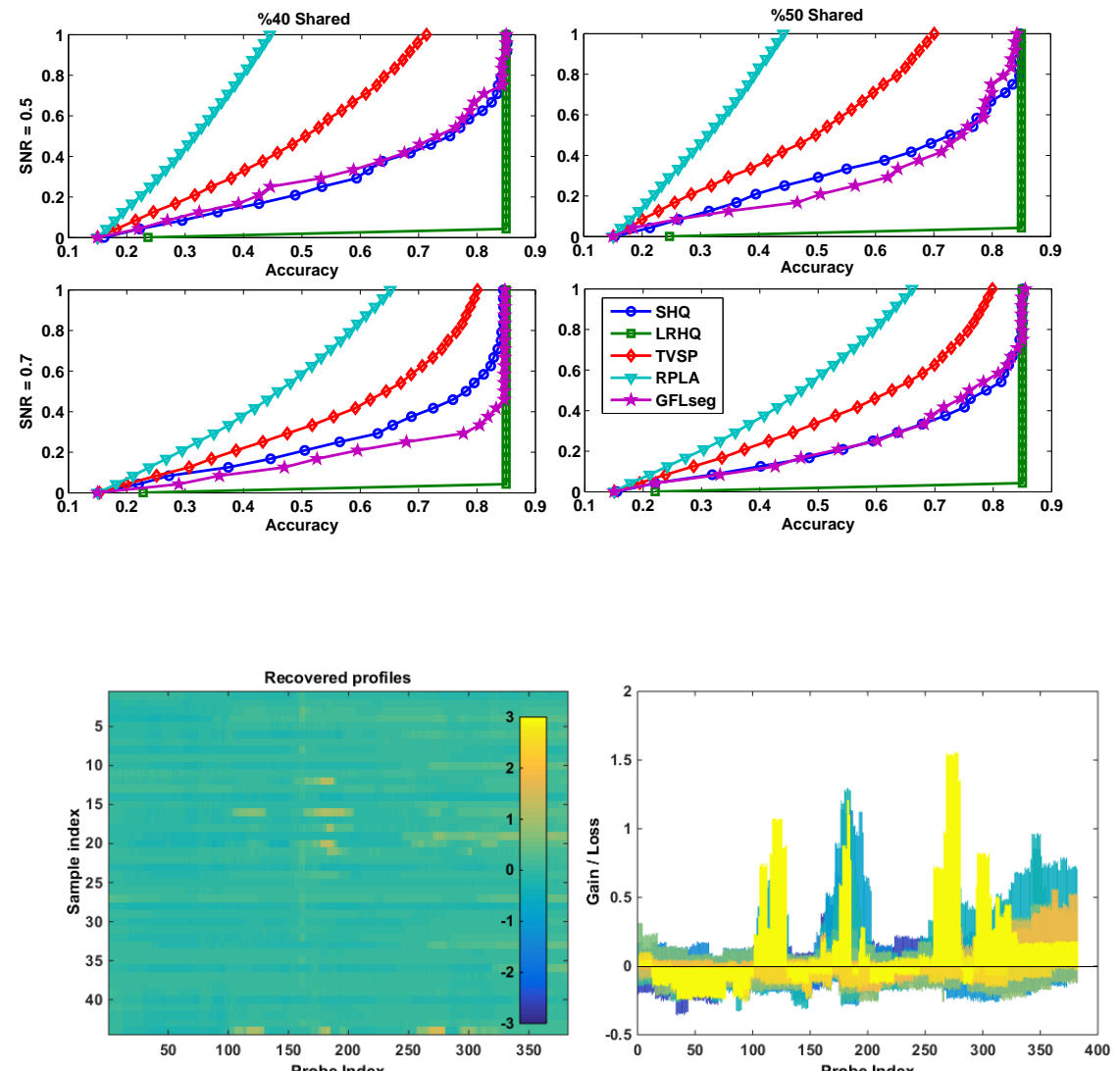

(a) LRHQ on Pollack et al. data set (32)
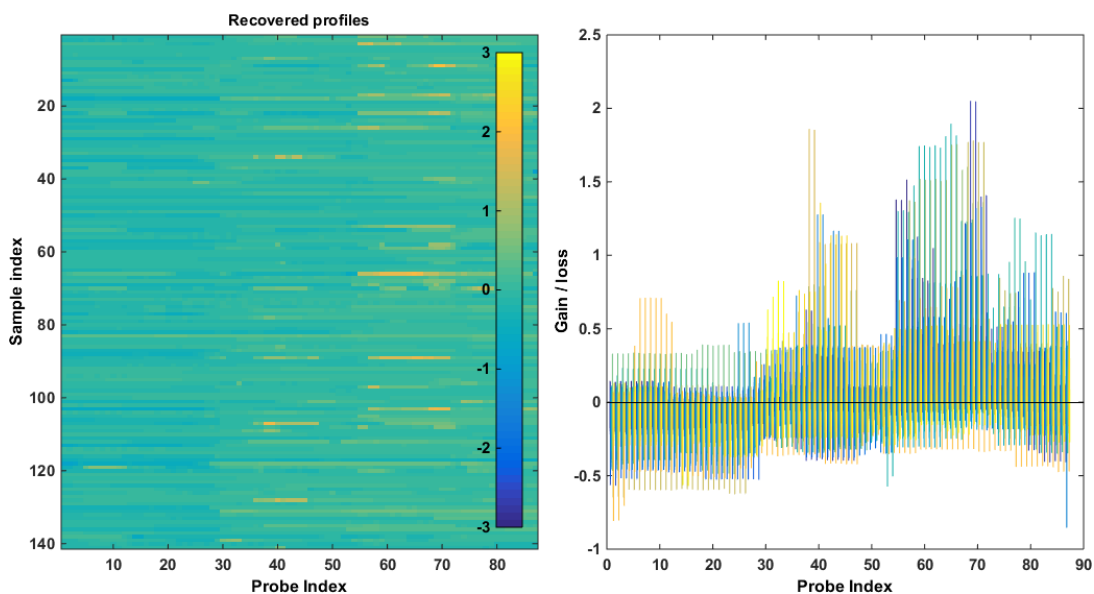

(b) SHQ on Chin et al. data set (33)

Figure 3: Heat and bar diagrams for real data sets. (a) Performing LRHQ on the data set introduced in [32] (b) Performing SHQ on the data set introduced in [33]. 

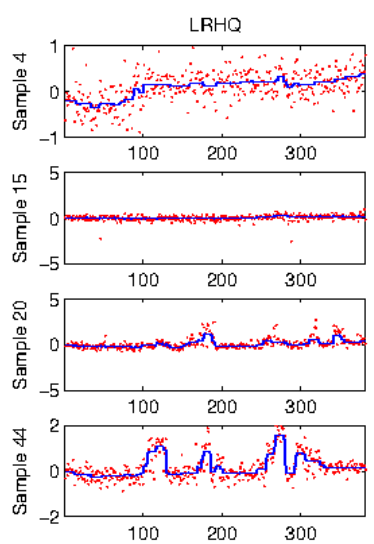
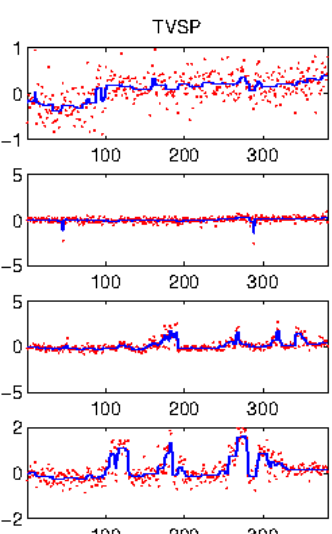

$100 \quad 200 \quad 300$
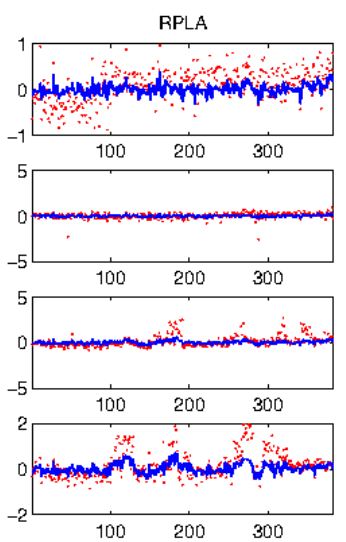
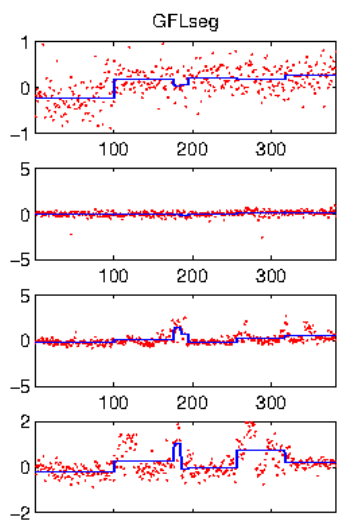

$100 \quad 200 \quad 300$

Figure 4: Array CGH profile of 4 chromosomes of the breast cancer data set (32). Panels in the same rows show the result of the same chromosome with different methods.

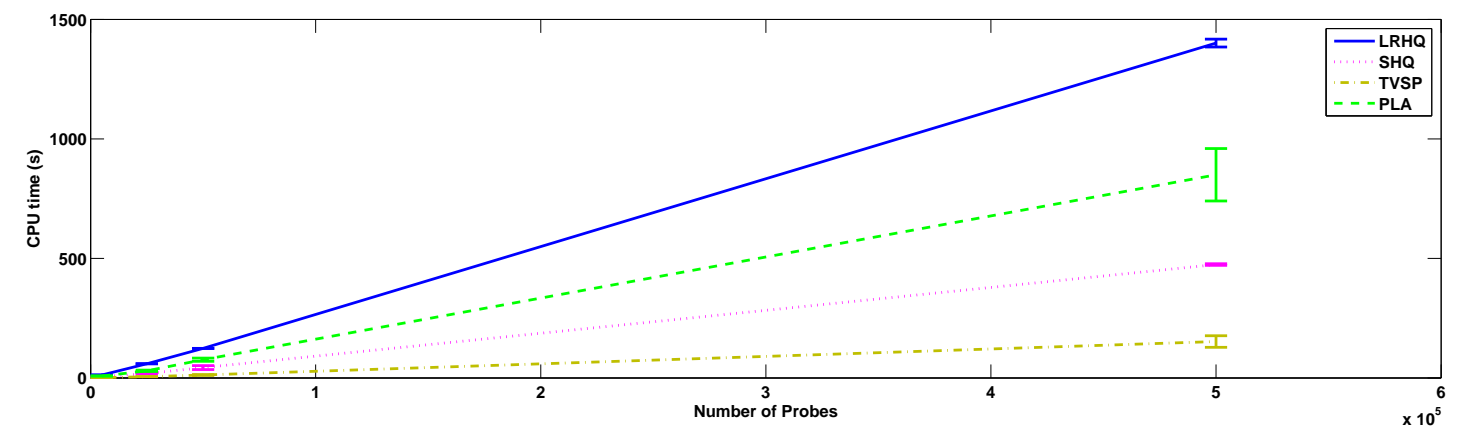

Figure 5: CPU time versus number of probes on simulated data with different numbers of probes. The number of samples is 100 . For each number of probes, each method is performed twice and the standrad deviation is reported. 
Table 1: Different M-estimators and their minimization functions $\delta($.$) relevant to the additive form of HQ. \alpha$ is a positive constant.

\begin{tabular}{|c|c|c|c|c|c|}
\hline estimators & $11-12$ & fair & log-cosh & Welsch & Huber \\
\hline loss function & $\sqrt{\alpha+\frac{t^{2}}{\sigma^{2}}}-1$ & $\frac{|t|}{\alpha}-\log \left(1+\frac{|t|}{\alpha}\right.$ & $\log (\cosh (\alpha t))$ & $1-\exp \left(-\frac{t^{2}}{\sigma^{2}}\right)$ & $\left\{\begin{array}{l}\frac{t^{2}}{2} \quad|t| \leq \lambda \\
\lambda|t|-\frac{\lambda^{2}}{2} \quad|t|>\lambda\end{array}\right.$ \\
\hline Additive form $\delta(t)$ & $t-\frac{t}{\sqrt{\alpha+\frac{t^{2}}{\sigma^{2}}}}$ & $t-\frac{t}{\alpha(\alpha+|t|)}$ & $t-\alpha \tanh (\alpha t)$ & $t-t \exp \left(-\frac{t^{2}}{\sigma^{2}}\right)$ & $\begin{cases}0 & |t| \leq \lambda \\
t-\lambda \quad \operatorname{sign}(t)|t|>\lambda\end{cases}$ \\
\hline
\end{tabular}

Table 2: Probability density functions (PDF) and their parameters utilized as an additive noise to simulated data. For generating noise, the parameters are randomly selected in the range tabulated in the last row.

\begin{tabular}{l|ccccc} 
distribution & Laplace & Gamma & Beta & Uniform & Exponential \\
\hline PDF & $f_{\text {Lap }}(\mu, \sigma)=\frac{1}{2 \sigma} e^{-\frac{|x-\mu|}{\sigma}}$ & $f_{\gamma}(k, \theta)=\frac{x^{k-1} e^{-\frac{x}{\theta}}}{\theta^{k} \Gamma(k)}$ & $f_{\beta}(\alpha, \beta)=\frac{x^{\alpha-1}(1-x)^{\beta-1}}{B(\alpha, \beta)}$ & $\left\{\begin{array}{ccc}\frac{1}{b-a} & a<x<b & \\
0 & \text { otherwise }\end{array}\right.$ & $\lambda e^{-\lambda x}$ \\
\hline settings & $\mu \in[0,5], \sigma \in[1,4]$ & $k \in[1,5], \theta \in[1,2]$ & $\alpha, \beta \in[0.5,5]$ & $a, b \in[3,-3]$ & $\lambda \in[0.5,2]$
\end{tabular}

